\title{
Probabilistic Prediction of Increased Intracranial Pressure in Patients With Severe Traumatic Brain Injury
}

Priyantha Wijayatunga

Umeå University

Lars-Owe D Koskinen

Umeå University

Nina Sundström ( $\square$ nina.sundstrom@umu.se )

Umeå University

\section{Research Article}

Keywords: Intensive care, Traumatic brain injury, Intracranial pressure, Time series data, Dynamic variation, Prediction accuracy

Posted Date: September 3rd, 2021

DOl: https://doi.org/10.21203/rs.3.rs-847403/v1

License: (a) (1) This work is licensed under a Creative Commons Attribution 4.0 International License. Read Full License 
1 Probabilistic prediction of increased intracranial pressure in

2 patients with severe traumatic brain injury

3

4 Priyantha Wijayatunga ${ }^{1,3}$, Lars-Owe D Koskinen ${ }^{2}$, Nina Sundström ${ }^{3}$

5

$6 \quad{ }^{1}$ Department of Statistics, Umeå University, Umeå, Sweden

$7 \quad{ }^{2}$ Department of Clinical Science - Neurosciences, Umeå University, Umeå, Sweden

$8{ }^{3}$ Department of Radiation Sciences, Radiation Physics, Biomedical Engineering, Umeå

9 University, Umeå, Sweden

10

12 ORCID

13 First author: (1)https://orcid.org/0000-0003-1654-9148

14 Second Author: https://orcid.org/0000-0003-3528-8502

15

16

17

21 Corresponding Author:

22 Nina Sundström, MsEP, $\mathrm{PhD}$

23 Department of Radiation Sciences, Radiation Physics, Biomedical Engineering, Umeå

24 University, Umeå, Sweden

25 Tel: +46703384482

26 E-mail: nina.sundstrom@umu.se 


\section{Abstract}

28 Background: Traumatic brain injury (TBI) causes temporary or permanent alteration in

29 brain functions. Generally, at intensive care units (ICU), intracranial pressure (ICP) is

30 monitored and treated to avoid increases in ICP with associated secondary insults and

31 poor clinical outcome. The aim of this study was to develop and evaluate a model which

32 could predict future ICP levels of individual patients during their treatment in the ICU,

33 and thus help the treating clinician to take proper actions before secondary injuries

34 occure.

35 Methods: A simple, explainable, probabilistic Markov model was developed for the

36 prediction task of ICP $\geq 20 \mathrm{mmHg}$. Predictions were made for consecutive 10-minute

37 intervals during the following hour, based on the preceding hour of ICP data. An easily

38 implementable enhancement method was also developed and applied to compensate for

39 imbalance in the data. The model was evaluated in a randomized and leave-one-out

40 fashion on data from 29 patients with severe TBI.

41 Results: With random data selection from all patients (80/20\% training/testing) and

42 including the new enhancement method, the specificity of the model was high (93.9-95.0)

43 and the sensitivity was good to high (72.7-87.1). Levels were similar (specificity 90.1-

$44 \quad 95.3$ and sensitivity 73.0-88.7) when the model was trained by the leave-one-out method

45 and evaluated on individual subjects.

46 Conclusion: The new model predicted increased levels of ICP in a reliable manner and

47 the enhancement method to compensate for imbalanced data further improved the

48 predictions. This lays the foundation for development of a bedside warning system

49 designed to proactively avoid increased ICP levels in patients with severe TBI. Further 
50 advantages are the straightforward expandability of the model, enabling inclusion of

51 other time series data and/or static parameters, making future studies of predictive

52 strength based on combinations of physiological data possible. Next step is to evaluate

53 the model on a larger patient material and to include other parameters apart from ICP.

58 Keywords: Intensive care, Traumatic brain injury, Intracranial pressure, Time series

59 data, Dynamic variation, Prediction accuracy

\section{$61 \quad$ Introduction}

62 Traumatic brain injury (TBI) is a type of head injury that causes temporary or permanent

63 alteration in brain functions. It is one of the major health problems currently worldwide.

64 Some of the main causes of TBI are automobile and sports accidents and falls (1). The

65 correct identification of clinical state of patients suffering severe TBI is often crucial for

66 subsequent medical treatments and for reduction of secondary injuries following the

67 initial insult. One such secondary injury is elevated levels of intracranial pressure (ICP)

68 which may result in decreased microcirculation, cerebral ischemia and infarcts (2).

69 Monitoring of ICP is recommended in patient with severe TBI with a Glasgow Coma

70 Scale score of 3-8 from the time of arrival to the intensive care unit (ICU) until the

71 clinical state improves, or in worst case, until death occurs $(3,4)$. An approximate mean

72 treatment time in the ICU is two weeks. Consensus guidelines from the Brain Traumatic

73 Foundation (5), state that a critical threshold for which treatment action should be

74 initiated to maintain ICP below is $22 \mathrm{mmHg}$, since levels above this are associated with 
75 poor clinical outcome. However, a limit of $20 \mathrm{mmHg}$ is also common in clinical practice,

76 including at our own hospital, and this limit was considered in the current study $(6,7)$.

78 During treatment in the ICU, it is difficult to know in which patient's the clinical state

79 will remain at a clinically stable level and in which one's ICP will increase rapidly to

80 dangerously high levels. Thus, the possibility to predict future increases in ICP, and treat

81 them to avoid additional pressure load on the brain and intracranial skull environment,

82 would be clinically very valuable. Further, it is of utmost importance for the clinician to

83 act before a secondary negative event is established.

85 Apart from ICP, the setup for multimodal monitoring in the ICU generally includes an

86 extensive battery of other high frequency physiological parameters such as mean arterial

87 blood pressure (ABP), cerebral perfusion pressure (CPP), echocardiography,

88 oxygenation, temperature, and respiration. However, this kind of extended monitoring is

89 not feasible in every part of the world, mainly due to lack of resources. Also, although the

90 information generated from multimodal monitoring equipment aids in the treatment

91 process in the ICU, it is generally too complex and diverse to be fully understood in real

92 time by the treating clinician (8). Thus, computational aids based on simple and relatively

93 easily obtainable data would be advantageous, but the possibility of incorporating richer

94 data streams as well opens for even better prediction models and further understanding of

95 the physiological processes involved during treatment in the ICU.

96 
97 During the last decades, computational power and methods based on artificial intelligence

98 and machine learning have increased tremendously. It is anticipated that these statistical

99 predictive methods will have a profound impact on health care in general, and on the

100 treatment in the ICU in particular, since the management is data-intense and often based

101 on multimodal monitoring $(9,10)$. In a recent review, it was shown that out of the 258

102 papers applying some kind of machine learning method to data routinely collected in the

103 ICU, the most common applications were predicting complications (29.8\%), predicting

104 mortality (27.1\%), improving prognostic models (16.7\%) and classifying sub-populations

$105(11.2 \%)(11)$. Thus, there is a need for models that can predict adverse events in real-time

106 during patient monitoring.

108 A model for ICP prediction in patients with TBI treated in the ICU has previously been

109 presented (9). This model predicted the occurrence of episodes (i.e., at least 10

110 consecutive minutes) with ICP $\geq 30 \mathrm{mmHg}$ with good accuracy. According to the

111 recommendations from the Brain Traumatic Foundation and the clinical guidelines at

112 Umeå University hospital, it would be preferable to have a system set out to warn already

113 at ICP > $20 \mathrm{mmHg}$. Also, the general "dose" regarding increased ICP has been shown to

114 correlate with clinical outcome (12-14), and mean ICP over 10-minute intervals, as

115 opposed to continuous values during the same period, has the potential to reflect this

116 burden while still being resilient to transient phenomena such as coughing.

117 A computational framework has also been proposed to predict prolonged intracranial

118 hypertension based on morphological waveform features computed from ICP variations

119 (15). Here, ICP > $20 \mathrm{mmHg}$ for longer than 5 minutes was the prediction target.

120 However, predictions were only made for time-to-onset between zero and 10 minutes,

121 and the areas under the curve and sensitivity need further improvement. Currently

122 popular machine learning methods, such as lasso regression, random forest models and 
123 support vector machines and traditional statistical methods, such as autoregression

124 models, have also been applied to the problem of ICP prediction (16). Their clinical

125 applicability needs to be further investigated, however, and focused on the clinically

126 more important problem of predicting dangerously high ICP levels as opposed to ICP

127 prediction in general.

128

129 This paper presents a model which addresses these issues in a computationally feasible

130 and simple manner. Furthermore, our model is probabilistic in nature, thus enabling

131 incorporation of many types of clinical information, and resulting in explainable outputs.

132

133 The aim of this study was to develop a probabilistic tool with explainable outputs to

134 predict future ICP levels of individual TBI patients in the ICU within the next hour to

135 come. The model should be based on ICP alone, as to be applicable in most ICU

136 facilities, but expandable to incorporate other time series and static patient information as

137 well. This enables future investigation of the predictive strength of combinations of

138 several physiological variables.

139

140 Methods

141

142 Patients

143 The probabilistic model presented and evaluated in this paper is based on ICP time series

144 data collected from 29 patients suffering from severe TBI and treated with neuro-

145 intensive care at Umeå University Hospital. Patient characteristics are described in Table

146 1. All patients were prospectively recruited from January 2015 to December 2017. 
147 Inclusion criteria were persons of all ages with a clinical diagnosis of severe TBI and an

148 indication for CT that presented to the hospital within $24 \mathrm{~h}$ of injury. Informed consent

149 was obtained according to local and national requirements. Patients with severe

150 preexisting neurological disorders that would confound outcome assessments were

151 excluded. All patients were treated by an ICP oriented regimen referred to as a modified

152 version of the Lund concept (17). This approach intends to keep ICP below $20 \mathrm{mmHg}$

153 and in some cases allowing CPP down to $50 \mathrm{mmHg}$, to avoid the negative effects of

154 pressor drugs and hypervolemia. CPP is generally not treated in order to exceed 60

$155 \mathrm{mmHg}$.

158 Table 1: Patient Characteristics.

\begin{tabular}{|l|c|}
\hline Variable & Value \\
\hline Median age in years (range) & $56(20-80)$ \\
\hline Sex [No. female/male] & $7 / 22$ \\
\hline Total monitoring time [h] & 4018 \\
\hline Mean (SD) monitoring time per patient [h] & $135.7(29.0)$ \\
\hline Mean (SD) ICP for all patients [mmHg] & $14.6(4.2)$ \\
\hline 6-months mortality [No. (\%)] & $4(14)$ \\
\hline 12-months mortality [No. (\%)] & $5(17)$ \\
\hline
\end{tabular}

159

160 
161 Time series data were collected by the Moberg CNS unit (Moberg Research, Inc.,

162 Ambler) as part of the CENTER-TBI study (18) and an ongoing local study. Sampling

163 frequency was $125 \mathrm{~Hz}$, which was rescaled to minute values. A software developed in

164 Matlab (Matlab 2019b, The MathWorks Inc, Natick, MA, USA) was applied to identify

165 all artifacts lasting longer than three seconds (2.5\%). In total, the model building and

166 testing were based on 4018 hours of ICP recordings. The limit for dangerously high ICP

167 levels was set to $20 \mathrm{mmHg}$, i.e., ICP $<20 \mathrm{mmHg}$ was classified as "1" (normal) and ICP

$168 \geq 20 \mathrm{mmHg}$ was classified as " 2 " (severe). Of the time series data, $81.7 \%$ were found to

169 be $<20 \mathrm{mmHg}$ (i.e., "ICP=1") and $18.3 \%$ were $\geq 20 \mathrm{mmHg}$ ("ICP=2"). Future ICP levels

170 were predicted for six continuous 10-minute intervals in the next hour from the present

171 time.

172

\section{Ethical approval}

174 The study was approved by the Ethics Review Authority in Umeå (2014/1473-31/4,

175 2011-256-31M, 00-175 \& 2013-43-31M) and followed the World Medical Association

176 Declaration of Helsinki.

\section{Model description}

179 A probabilistic model for the prediction of future ICP levels of individual patients was

180 developed. The model is generalizable to any number of predictor variables but can be

181 simplified when predictions are to be based on ICP data streams alone. The model is a

182 more general dynamic version of the so-called naïve Bayes model (19), i.e., both the

183 predictors and the predicted variables can be time series, although some of the predictors

184 can be static random variables as well. 
186 Let $X_{i: t}$ denote the observation at time $t$ (discrete, for simplicity) of the $i^{\text {th }}$ time series, for

$187 t=1,2,3, \ldots$ and $i=1, \ldots, p$. Here $p \geq 1$ is the number of time series denoted by

$188\left\{X_{1: t}\right\}, \ldots,\left\{X_{p: t}\right\}$. Assume that there are no long-term trends in any of the time series. The

189 model should predict, without loss of generality, all $m$ number of time steps into the

190 future from current time $t$ of the $1^{\text {st }}$ time series, i.e., $\left(X_{1: t+1}, \ldots, X_{1: t+m}\right)$. For the model to

191 make independent predictions for each of these time steps, it is defined as:

192

$$
P\left(X_{1: t+k} \mid X_{1:\left[t-d_{1}, t\right]}, \ldots, X_{p:\left[t-d_{p}, t\right]}\right)=\frac{P\left(X_{1: t+k}\right) \prod_{i=1}^{p} P\left(X_{i:\left[t-d_{i}, t\right]} \mid X_{1: t+k}\right)}{\sum_{X_{1: t+k}^{\prime}} P\left(X_{1: t+k}^{\prime}\right) \prod_{i=1}^{p} P\left(X_{i:\left[t-d_{i}, t\right]} \mid X_{1: t+k}^{\prime}\right)}
$$

194 where $k=1, \ldots, m$ and integer $d_{i}>0$ for $i=1, \ldots, p$. Here

$195 X_{i:\left[t-d_{i}, t\right]}=\left(X_{i: t-d_{i}}, X_{i: t-d_{i}+1}, \ldots, X_{i: t}\right)$ is the history of the $i^{\text {th }}$ time series $d_{i}$ number of

196 time steps back from the present time $t$, for $i=1, \ldots, p$. The model gives conditional

197 probability of the value of the $k^{\text {th }}$ time step of the $1^{\text {st }}$ series, given a finite history of each

198 of the time series available.

200 In this study the simplest version of the above model, where $p=1$ for $d_{1}>1$, was

201 evaluated. When mean ICP at a desired time interval into the future, e.g., the $k^{\text {th }}$ time step

202 from the present time $t$, is predicted using only the history of ICP itself from the past time

$203\left(t-d_{1}\right)$ until the present time, it is obtained as

204

205

$$
x_{1: t+k}^{*}=\operatorname{ArgMax}_{x_{1: t+k}} p\left(x_{1: t+k} \mid x_{1:\left[t-d_{1}, t\right]}\right) .
$$


207 Note that when $k=1$, the average ICP value for the first 10-minute interval from the

208 present time is predicted, when $k=2$, the second 10-minute interval is predicted and so

209 on.

210

211 Prediction enhancement

212 To achieve a prediction enhancement favoring more accurate predictions of instances of

$213 \mathrm{ICP} \geq 20 \mathrm{mmHg}$ (ICP classified as “2”), the $k^{\text {th }}$-time step prediction was obtained as

$215 x_{1: t+k}^{*}=\operatorname{ArgMax}_{x_{1: t+k}}\left\{\begin{array}{c}\alpha p\left(x_{1: t+k}=1 \mid x_{1:\left[t-d_{1}, t\right]}\right) \\ p\left(x_{1: t+k}=2 \mid x_{1:\left[t-d_{1}, t\right]}\right)\end{array}\right\}$

217 where $0<\alpha \leq 1$. Here, $\alpha$ acts as a "balancing factor" to compensate for the imbalance

218 of the data. E.g., when $\alpha=0.65$ is selected, if we have $p\left(x_{1: t+k}=1 \mid x_{1:\left[t-d_{1}, t\right]}\right)=0.6$

219 and therefore $p\left(x_{1: t+k}=2 \mid x_{1:\left[t-d_{1}, t\right]}\right)=0.4$, then the prediction is $x_{1: t+k}^{*}=2$, since

$2200.65 * 0.6=0.39<0.40$. The selection of $\alpha$ was based on the equation:

$$
T=A f_{1}(\alpha)+w(1-A) f_{2}(\alpha)
$$

223 where $A$ is the fraction of $I C P=1, f_{i}(\alpha)$ is the gain in prediction accuracy for $I C P=i$

224 where $i=1,2$, due to $\alpha$, and $w \geq 1$ is the weight of $I C P=2$ compared to that of $I C P=1$. For

225 obtaining enhanced predictions favoring accuracy in $\mathrm{ICP}=2$ as opposed to $\mathrm{ICP}=1, w=2$

226 and $\alpha=0.60$ in this study. 
229 All data were divided into 10-minute intervals. Mean ICP of each interval was defined as

230 the response value. The preceding hour of data (i.e., six 10-minute intervals) were taken

231 as the predictor values for that specific response value. These predictors were discretized

232 into three levels based on their third quartile of $\operatorname{ICP}\left(\mathrm{ICP}_{3 \mathrm{Q}}\right)$ such that $1: \mathrm{ICP}_{3 \mathrm{Q}}<15$

$233 \mathrm{mmHg}, 2: 15 \leq \mathrm{ICP}_{3 \mathrm{Q}}<20$ and 3: $\mathrm{ICP}_{3 \mathrm{Q}} \geq 20 \mathrm{mmHg}$, respectively.

\section{Evaluation of the probabilistic model for ICP prediction}

237 Prediction accuracy was evaluated based on a random selection of 10-minute intervals

238 from all patients. This was done for all six intervals of the next hour from the present.

239 That is, for each patient, $80 \%$ of the 10-minute intervals were randomly selected for

240 training the model while the remaining $20 \%$ were used as test data. Mean prediction

241 accuracies for each 10-minute interval along with their non-parametric bootstrap

242 confidence intervals (CI) were calculated. In a similar manner, the mean prediction

243 accuracies for individual patients were also evaluated. Here, the model was based on

244 continuous ICP time series data from all but one patient, for which the predictions were

245 evaluated.

\section{Statistical analyses}

248 All model building, training and statistical analyses were performed using the free

249 software R (20) on an Apple MacBook Pro Retina (Processor: 2.5 GHz Quad-core Intel

250 Core i7, Memory: 16BG, 1600MHz with macOS Big Sur version 11.3.1). 


\section{Results}

253 Figure 1 shows the distribution of mean ICP over 10-minute intervals for three selected 254 subjects and for all 29 subjects, respectively. The highly imbalanced nature of the data, 255 regarding the prediction threshold of $20 \mathrm{mmHg}$, is clearly displayed.
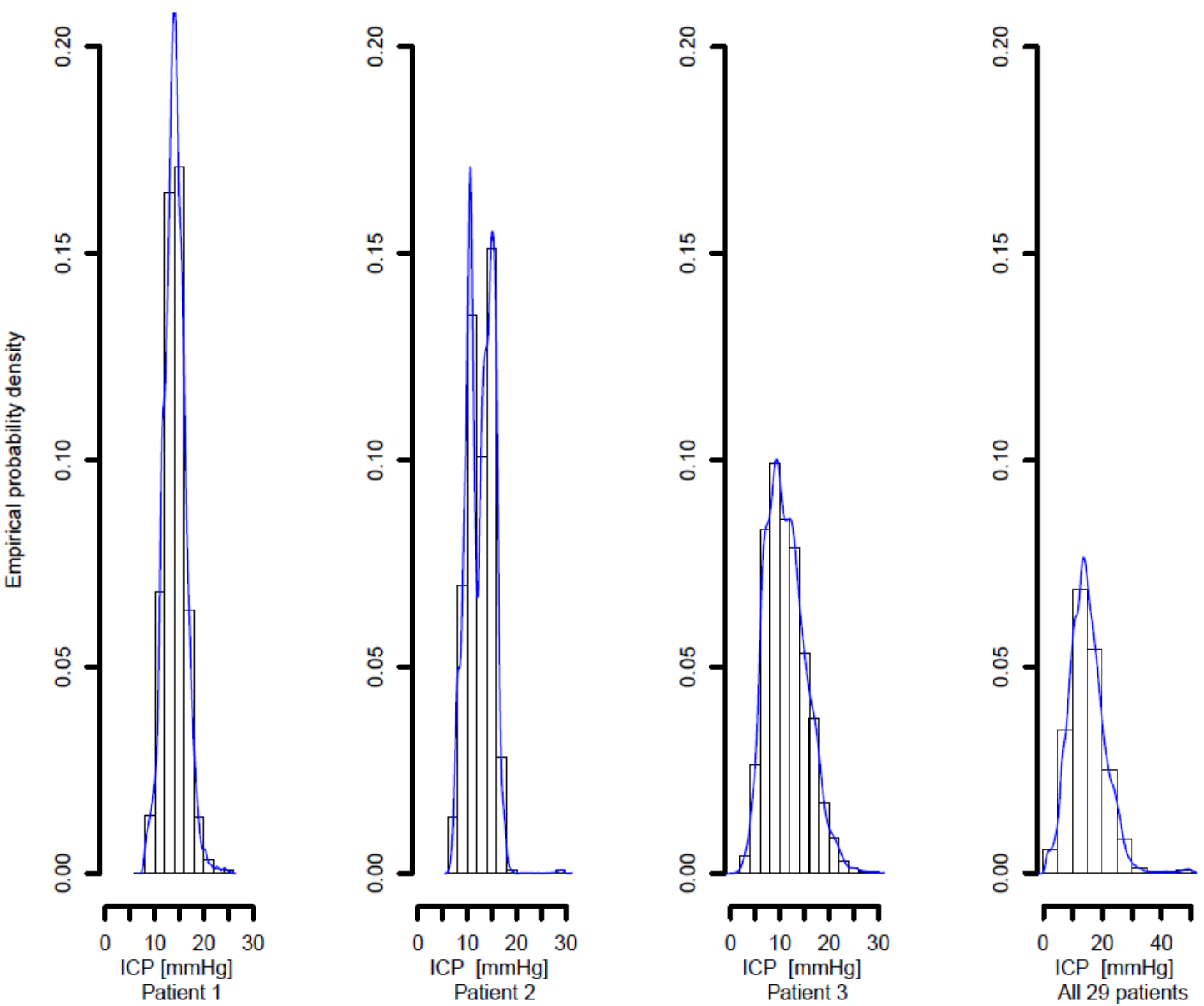

257 Figure 1: Probability density of ICP for three typical patients monitored and treated in the

258 ICU (first three distributions), and during the total monitoring time for all 29 patients

259 (rightmost distribution). 
Table 2 shows the mean prediction accuracies and $95 \%$ bootstrap CI for test sets of

263 randomly selected instances each 10-minute interval in the next hour. Table 3 shows the

264 weighted mean and range for the prediction accuracies of individual patients. The

265 weights were based on the proportion of ICP $=1$ and ICP $=2$ for each patient, respectively.

266

268 Table 2. Prediction accuracy (non-parametric bootstrap 95\% CI)

\begin{tabular}{|c|c|c|c|c|c|}
\hline & & Specificity (o) & rue ICP="1" & Sensitivity (\%) & rue ICP="2 \\
\hline & $\begin{array}{c}\text { 10- } \\
\text { minute } \\
\text { interval }\end{array}$ & General $^{\mathrm{a}}$ & Enhanced $^{b}$ & General $^{\mathbf{a}}$ & Enhanced $^{b}$ \\
\hline & First & $\begin{array}{c}96.0 \\
(95.8 ; 96.2)\end{array}$ & $\begin{array}{c}95.0 \\
(94.9 ; 95.1)\end{array}$ & $\begin{array}{c}85.4 \\
(83.6 ; 85.1)\end{array}$ & $\begin{array}{c}87.1 \\
(86.3 ; 87.7)\end{array}$ \\
\hline e & Second & $\begin{array}{c}96.1 \\
(95.9 ; 96.3)\end{array}$ & $\begin{array}{c}94.7 \\
(94.5 ; 94.9)\end{array}$ & $\begin{array}{c}74.7 \\
(73.5 ; 75.8)\end{array}$ & $\begin{array}{c}80.6 \\
(80.1 ; 81.2)\end{array}$ \\
\hline 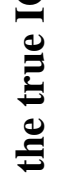 & Third & $\begin{array}{c}95.6 \\
(95.5 ; 95.7)\end{array}$ & $\begin{array}{c}93.8 \\
(93.6 ; 93.9)\end{array}$ & $\begin{array}{c}72.1 \\
(69.8 ; 75.6)\end{array}$ & $\begin{array}{c}75.7 \\
(74.8 ; 76.7)\end{array}$ \\
\hline 凷: & Fourth & $\begin{array}{c}95.5 \\
(95.3 ; 95.7)\end{array}$ & $\begin{array}{c}93.0 \\
(92.9 ; 93.2)\end{array}$ & $\begin{array}{c}67.4 \\
(66.3 ; 68.4)\end{array}$ & $\begin{array}{c}75.0 \\
(74.3 ; 75.9)\end{array}$ \\
\hline 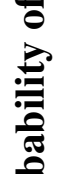 & Fifth & $\begin{array}{c}95.2 \\
(95.0 ; 95.5)\end{array}$ & $\begin{array}{c}93.3 \\
(93.0 ; 93.4)\end{array}$ & $\begin{array}{c}67.3 \\
(66.0 ; 68.6)\end{array}$ & $\begin{array}{c}73.0 \\
(72.4 ; 73.6)\end{array}$ \\
\hline 2 & Sixth & $\begin{array}{c}95.1 \\
(94.9 ; 95.2)\end{array}$ & $\begin{array}{c}93.9 \\
(93.6 ; 94.1)\end{array}$ & $\begin{array}{c}67.0 \\
(66.3 ; 67.8)\end{array}$ & $\begin{array}{c}72.7 \\
(72.1 ; 73.4)\end{array}$ \\
\hline
\end{tabular}


$269{ }^{a}$ General predictions based on maximum likelihood estimation. ${ }^{b}$ Enhanced predictions

270 obtained with $\alpha=0.60$.

271

272 Table 3. Enhanced weighted mean prediction accuracy for individual patients (range of

273 variation), $\alpha=0.60$.

\begin{tabular}{|c|c|c|c|c|c|c|c|}
\hline & $\begin{array}{l}\text { 10-minute } \\
\text { interval }\end{array}$ & First & Second & Third & Fourth & Fifth & Sixth \\
\hline Prediction & $\begin{array}{c}\text { True } \\
\text { ICP = "1" }\end{array}$ & $\begin{array}{c}95.3 \\
(55 ; 100)\end{array}$ & $\begin{array}{c}93.0 \\
(33 ; 100)\end{array}$ & $\begin{array}{c}92.2 \\
(33 ; 100)\end{array}$ & $\begin{array}{c}91.9 \\
(25 ; 100)\end{array}$ & $\begin{array}{c}90.4 \\
(25 ; 100)\end{array}$ & $\begin{array}{c}90.1 \\
(25 ; 100)\end{array}$ \\
\hline$(\%)$ & $\begin{array}{c}\text { True } \\
\text { ICP = "2" }\end{array}$ & $\begin{array}{c}88.7 \\
(21 ; 97)\end{array}$ & $\begin{array}{c}83.2 \\
(10 ; 95)\end{array}$ & $\begin{array}{c}79.6 \\
(10 ; 97)\end{array}$ & $\begin{array}{c}78.4 \\
(10 ; 97)\end{array}$ & $\begin{array}{c}75.4 \\
(10 ; 96)\end{array}$ & $\begin{array}{c}73.0 \\
(10 ; 94)\end{array}$ \\
\hline
\end{tabular}

274

\section{Discussion}

276 This paper describes and evaluates a simple and explainable probabilistic model for

277 prediction of ICP levels of individual patients with severe TBI during treatment in the

278 ICU. Predictions were made for the next hour to come, based only on the past hour of

279 ICP variation of that patient. A model for prediction enhancement was also presented and

280 evaluated, showing that a simplistic method for data weighting could compensate for

281 imbalanced data and considerably improve prediction accuracy.

283 Prediction accuracy and clinical applicability 
284 For the prediction task ICP $\geq 20 \mathrm{mmHg}$, the model presented in this study had a very

285 high specificity (93.9-95.0) and a good to high sensitivity (72.7-87.1). Results were

286 similar (specificity 90.1-95.3 and sensitivity 73.0-88.7) when the model was trained

287 based on the leave-one-out method and evaluated on individual patients, which is

288 important since this will be the case in the clinical situation. The wide ranges of variation

289 when predictions were made for individual patients uninterruptedly are a result of the

290 very few instances of severe ICP in some subjects. Low prediction accuracy for rare

291 occasions is a very common aspect of most prediction models. For both models the

292 prediction accuracy decrease the further away from the current time point the prediction

293 was made. This is reasonable since the near past is likely to be more informative of the

294 future, but this shows the need to determine a threshold between the time needed to take

295 necessary actions in the ICU to avoid secondary insults, as opposed to having too low

296 prediction accuracy for time periods further ahead.

298 Güiza et.al. were the first to build a model to predict future ICP (9). They used multiple 299 logistic regression and Gaussian processes to predict continuous ICP $>30 \mathrm{mmHg}$ for at

300 least 10 minutes 30 minutes in advance based on 4 hours of past ICP and ABP. Their

301 model had a classification accuracy of $77 \%$, a sensitivity of $82 \%$ and a specificity of $75 \%$

302 in the first validation cohort. The model was later validated on two other cohorts of adult

303 TBI patients; 151 subjects part of a multicenter study including five centers (21) and 257

304 other subjects recruited as part of the CENTER-TBI high-resolution ICU monitoring

305 cohort (22). The precision varies somewhat between the cohorts (sensitivity/specificity 

$70 / 90 \%$ and $83 / 91 \%$ respectively) but are overall in the same range as the ones found

307 with our model.

309 Recently, an online machine learning and signal processing framework that forecasts

310 onsets of acute intracranial hypertension up to 8 hours in advance using waveforms of

311 different physiological signals was introduced (23). Their model had a sensitivity of $90 \%$

312 but the precision was only $30 \%$ based on data from a waveform database. The study

313 showed the importance of information contained in high-frequency waveforms in

314 neurological signals, that could motivate future studies on pre-hypertensive patterns and

315 the design of new alarm algorithms for critical events in TBI patients.

317 There are also other model applications that did not reach as high prediction accuracy,

318 i.e., a prediction of prolonged intracranial hypertension based on morphological

319 waveform features computed from ICP variations (15). Here, ICP > $20 \mathrm{mmHg}$ was

320 predicted for time-to-onset between zero and 10 minutes with a sensitivity/specificity of

$32152 / 72 \%$ for predictions made six minutes ahead.

\section{Probabilistic model}

324 The conditional probabilities (parameters) of the current model are estimated with the

325 method of maximum likelihood, i.e., they have closed form solutions and no assumptions

326 of statistical models are needed. These kinds of estimates enable general predictions of

327 future events, as opposed to standard Bayesian methods such as maximum a posteriori

328 estimation where subjective opinions of the probabilities of different events are also 
329 needed, in addition to data. Discriminative parameter learning methods, such as

330 maximization of conditional likelihood (24) and minimization of prediction error

331 measures (defined by weighting predictions appropriately) (19) are also applicable to

332 obtain higher prediction accuracies. However, such methods enhance prediction

333 accuracies of all possible outcome levels, often resulting in only slight increases in

334 overall prediction accuracies at the expense of computationally intense algorithms. Here,

335 another advantage with the maximum likelihood method is that it enables enhancement of

336 the more important category of the response variable alone, and may be combined with

337 our suggested prediction enhancement method without unnecessary complexity or

338 computational burden.

340 When developing a prediction model, there is always a trade-off between sensitivity and

341 specificity. The level of the trade-off must be determined by the application at hand, and

342 what kind of error is "tolerable" in the specific clinical situation. When monitoring ICP in

343 patients with TBI, time frames with severely high ICP levels are much less frequent than

344 those with normal levels, e.g., due to the treatment regimen applied to avoid high ICP

345 levels. This results in highly imbalanced data with large amounts of "normal" and small

346 amounts of the "abnormal" levels that are set out to be predicted. When such data are

347 used for computational prediction model building, the prediction accuracies for minority

348 categories are often dominated by those of majority categories, resulting in a clinically

349 less valuable model. One strategy to avoid this problem in model building is either to

350 down sample the majority categories or up sample the minority categories when forming

351 the training data. However, this may introduce sampling bias into the training data, thus 
352 affecting the model explainability and accuracy. In this paper, a different strategy to

353 handle the imbalanced data was applied. This approach is directed towards increasing the

354 prediction accuracies of the minority categories which are clinically more important.

355 Based on clinical experience, the correct prediction of ICP $\geq 20 \mathrm{mmHg}$ was considered to

356 be twice as important ( $\mathrm{w}=2$ ) compared to erroneously predicting ICP $\geq 20 \mathrm{mmHg}$ when it

357 was truly $<20 \mathrm{mmHg}$, i.e., it was considered twice as imported to be alerted of a possible

358 pressure increase even though this might lead to some additional false alarms. Actions

359 that may be taken to prevent a dangerous ICP elevation include optimization of

360 homeostatic and respiratory parameters, positional change of the patient, CT investigation

361 to rule out space occupying lesions suitable for surgical intervention and treatment of

362 acute hydrocephalus in need of external ventricular drain.

363

364 The selection of $\alpha$ is crucial. It can be selected subjectively or objectively depending on

365 the application at hand. A possible method based on semi-objective criteria for total

366 prediction accuracy gain $T$ was implemented in this study. Note that generally, $f_{1}(\alpha) \leq 0$

367 and $f_{2}(\alpha) \geq 0$, e.g., one can define $f_{i}(0.60)$ as the difference between the mean

368 prediction accuracy for ICP=i with $\alpha=1$ and $\alpha=0.60$, for $i=1,2$. A mathematical

369 way to select a value of $\alpha$ is to maximize the value of $T$. However, any near-optimal

370 value chosen heuristically may work as well. In this study, $\alpha=0.60$ leads to

371 considerable enhancement of the prediction accuracy for ICP=2 at the expense of very

372 little loss of prediction accuracy for $\mathrm{ICP}=1$. Predicting $\mathrm{ICP}=2$ when the true value is

373 ICP=1 may also often be towards the end of a spell of true ICP value of "2". So, in

374 practice such an error may be handled rather easily. 


\section{General probabilistic model}

377 The general model was introduced to show that it is easy to combine many variables, e.g.,

378 not only several time series but also static observations such as sex, age, medical scores,

379 etc. into the prediction process to evaluate the predictive strength of different

380 combinations of variables. The possibility to include many predictors is one of the main

381 strengths of the proposed model, apart from its ease in implementation. Furthermore, this

382 model can be used in various ways, e.g., based on different time scales, different statistics

383 of the observations, etc. In this sense, it is a more flexible model than e.g., standard

384 autoregressive time series models or even traditional neural network models, where raw

385 observations are generally used as predictors. Time and frequency domain features and

386 use of waveforms can reveal complex dependencies which may be valuable for the

387 prediction task (25). Use of such features in our general model is straightforward.

388 However extensive modelling needs to be conducted to identify "best" feature variables.

389 Ideally, such experiments should include large data sets from several centers to enable

390 context independent models.

\section{Conclusion}

393 A new model for predicting ICP during ICU treatment, based on past ICP only, was

394 developed and evaluated. A new and easily implementable enhancement method, to

395 compensate for imbalanced data, was also presented. The prediction model is promising

396 for further development into a tool offering a proactive warning system to avoid

397 secondary insults and associated poor outcomes with regards to increases in ICP. The 
398 model can also be fully generalized for incorporation of other time series data or clinical

399 parameters, thus making future studies of various combination effects possible.

400

$401 \quad$ List of abbreviations

402 3Q: Third quartile

403 ABP: Arterial blood pressure

404 CI: Confidence interval

405 CPP: Cerebral perfusion pressure

406 ICP: Intracranial pressure

407 ICU: Intensive care unit

408 TBI: Traumatic brain injury

409

410

411

412 Declarations

413 Ethics approval and consent to participate

414 The study was approved by the Regional Ethical Review Board (2014/1473-31/4, 2011-

415 256-31M, 00-175 \& 2013-43-31M) and followed the World Medical Association

416 Declaration of Helsinki. Informed consent was obtained according to local and national

417 requirements.

418

419 Consent for publication 
$420 \quad$ Not applicable

421

422 Availability of data and materials

423 The datasets used and/or analysed during the current study are available from the

424 corresponding author on reasonable request.

425

426 Competing interests

427 The authors declare that they have no competing interests.

428

429 Funding Source

430 This study was financially supported by: Umeå clinical neuroscience research fund,

431 Umeå university, Regional agreement between Umeå University and Västerbotten

432 County Council on cooperation in field of medicine, odontology and health. The funding

433 body did not have any part in the design of the study, collection, analysis or interpretation

434 of data or in writing the manuscript.

435

436 Authors' contributions

437 All authors contributed to the study conception and design. L-O D K was responsible for

438 the data collection and PW and NS prepared the data for analysis. PW performed

439 statistical model development and analyses. The first draft of the manuscript was written

440 by PW and NS and all authors reviewed, edited and approved the final manuscript. 


\section{References}

446 1. Majdan M, Plancikova D, Brazinova A, Rusnak M, Nieboer D, Feigin V, et al. 447 Epidemiology of traumatic brain injuries in Europe: a cross-sectional analysis. Lancet 448 Public Health. 2016;1(2):e76-e83.

449 2. Donnelly J, Czosnyka M, Adams H, Cardim D, Kolias AG, Zeiler FA, et al.

450 Twenty-Five Years of Intracranial Pressure Monitoring After Severe Traumatic Brain

451 Injury: A Retrospective, Single-Center Analysis. Neurosurgery. 2019;85(1):E75-E82.

452 3. Stocchetti N, Picetti E, Berardino M, Buki A, Chesnut RM, Fountas KN, et al.

453 Clinical applications of intracranial pressure monitoring in traumatic brain injury : report

454 of the Milan consensus conference. Acta Neurochir (Wien). 2014;156(8):1615-22.

455 4. Brain Trauma F, American Association of Neurological S, Congress of

456 Neurological S, Joint Section on N, Critical Care AC, Bratton SL, et al. Guidelines for

457 the management of severe traumatic brain injury. VI. Indications for intracranial pressure

458 monitoring. J Neurotrauma. 2007;24 Suppl 1:S37-44.

459 5. Carney N, Totten AM, O'Reilly C, Ullman JS, Hawryluk GW, Bell MJ, et al.

460 Guidelines for the Management of Severe Traumatic Brain Injury, Fourth Edition.

461 Neurosurgery. 2017;80(1):6-15.

$462 \quad 6 . \quad$ Olivecrona M, Rodling-Wahlstrom M, Naredi S, Koskinen LO. Effective ICP

463 reduction by decompressive craniectomy in patients with severe traumatic brain injury

464 treated by an ICP-targeted therapy. J Neurotrauma. 2007;24(6):927-35.

465 7. Claassen J, Rahman SA, Huang Y, Frey HP, Schmidt JM, Albers D, et al. Causal

466 Structure of Brain Physiology after Brain Injury from Subarachnoid Hemorrhage. PLoS

467 One. 2016;11(4):e0149878.

$468 \quad 8$. Faltermeier R, Proescholdt MA, Bele S, Brawanski A. Parameter Optimization for

469 Selected Correlation Analysis of Intracranial Pathophysiology. Comput Math Methods

470 Med. 2015;2015:652030.

$4719 . \quad$ Guiza F, Depreitere B, Piper I, Van den Berghe G, Meyfroidt G. Novel methods

472 to predict increased intracranial pressure during intensive care and long-term neurologic

473 outcome after traumatic brain injury: development and validation in a multicenter dataset.

474 Crit Care Med. 2013;41(2):554-64.

475 10. Faltermeier R, Proescholdt MA, Bele S, Brawanski A. Windowed multitaper

476 correlation analysis of multimodal brain monitoring parameters. Comput Math Methods

477 Med. 2015;2015:124325.

478 11. Shillan D, Sterne JAC, Champneys A, Gibbison B. Use of machine learning to

479 analyse routinely collected intensive care unit data: a systematic review. Crit Care.

$480 \quad 2019 ; 23(1): 284$.

481 12. Vik A, Nag T, Fredriksli OA, Skandsen T, Moen KG, Schirmer-Mikalsen K, et al.

482 Relationship of "dose" of intracranial hypertension to outcome in severe traumatic brain

483 injury. J Neurosurg. 2008;109(4):678-84.

484 13. Sheth KN, Stein DM, Aarabi B, Hu P, Kufera JA, Scalea TM, et al. Intracranial

485 pressure dose and outcome in traumatic brain injury. Neurocrit Care. 2013;18(1):26-32. 

measurement of "pressure times time dose" of intracranial hypertension best predicts outcome after severe traumatic brain injury. J Trauma. 2010;69(1):110-8. 15. Scalzo F, Hamilton R, Asgari S, Kim S, Hu X. Intracranial hypertension prediction using extremely randomized decision trees. Med Eng Phys. 2012;34(8):105865. Forecasting in Children Using Dynamic Averaging of Time Series Data. Forecasting. 2019;1(1):47-58.

17. Koskinen LO, Olivecrona M, Grande PO. Severe traumatic brain injury management and clinical outcome using the Lund concept. Neuroscience. 2014;283:24555.

18. Maas AIR, Menon DK, Adelson PD, Andelic N, Bell MJ, Belli A, et al. Traumatic brain injury: integrated approaches to improve prevention, clinical care, and research. Lancet Neurol. 2017;16(12):987-1048.

501 19. Wijayatunga P, Mase, S. and Nakamura, M. . Appraisal of companies with

502 Bayesian networks. . International Journal of Business Intelligence and Data Mining. $503 \quad 2006 ; 1(3): 17$.

504 20. Team RC. R: A language and environment for statistical computing.: R

505 Foundation for Statistical Computing, Vienna, Austria; 2019.

506 21. Guiza F, Depreitere B, Piper I, Citerio G, Jorens PG, Maas A, et al. Early

507 Detection of Increased Intracranial Pressure Episodes in Traumatic Brain Injury: External

508 Validation in an Adult and in a Pediatric Cohort. Crit Care Med. 2017;45(3):e316-e20.

509 22. Carra G, Guiza F, Depreitere B, Meyfroidt G, Participants C-TH-RIS-S,

510 Investigators. Prediction model for intracranial hypertension demonstrates robust

511 performance during external validation on the CENTER-TBI dataset. Intensive Care Med.

$512 \quad 2021 ; 47(1): 124-6$.

513 23. Huser M, Kundig A, Karlen W, De Luca V, Jaggi M. Forecasting intracranial

514 hypertension using multi-scale waveform metrics. Physiol Meas. 2020;41(1):014001.

515 24. Grossman $\mathrm{DaD}, \mathrm{P}$. . Learning Bayesian network classifiers by maximizing

516 conditional likelihood. Proceedings of the 21st International Conference on Machine

517 Learning (ICML-2004). 2004:8.

518 25. Dai H, Jia X, Pahren L, Lee J, Foreman B. Intracranial Pressure Monitoring

519 Signals After Traumatic Brain Injury: A Narrative Overview and Conceptual Data

520 Science Framework. Front Neurol. 2020;11:959.

\section{Figure legends}

\section{$524 \quad$ Figure 1}


525 Probability density of ICP for three typical patients monitored and treated in the ICU

526 (first three distributions), and during the total monitoring time for all 29 patients

527 (rightmost distribution). 